\title{
Miopatías post anestésicas en equinos fina sangre de carrera
}

\author{
ANA CANALES G. ${ }^{1}$ y ADOLFO GODOY P. ${ }^{1}$
}

1 Hospital Clínico Veterinario de Equinos. Facultad de Ciencias Veterinarias y Pecuarias.

\begin{abstract}
POST-ANESTHESIA MYOPATHIES IN THOROUGHBRED HORSES

The Thoroughbred horses are athletes who are constantly subjected to high physical demands, often as a result bring disturbance of the musculoskeletal system. Many of these abnormalities require surgical treatment and therefore general anesthesia, like what happens with the affections of the upper airway from trauma and multiple lacerations. Given the anatomical and physiological characteristics typical of this species, there are several risks involved general anesthesia. The recumbency can eventually and depending on its duration, cause cardiorespiratory and muscular disorders that can compromise normal patient recovery. Muscle damage, which can generate serious myopathies necrosis, serious product from the pressure exerted by the mass and skeletal muscle, as also the commitment of muscle tissue hypoxia. It was observed that the dorsal recumbency is what causes most damage with respect to lateral recumbency and left lateral position produces less damage than the right. The degree of muscle damage be quantified through the study of serum enzymes CK, LDH, AST. Prevention of this type of disease goes through a history and feeding management of horses prior to undergo general anesthesia.

Key word: Anaesthesia, Myopathies, horses, serum enzymes.
\end{abstract}

\section{RESUMEN}

Los Equinos Fina Sangre de carrera son atletas que están constantemente sometidos a grandes demandas físicas, lo que a menudo se traduce en problemas del sistema musculo esquelético. Muchas de estas anomalidades requieren de un tratamiento quirúrgico y por lo tanto, de anestesia general, similar a lo que sucede con las afecciones de las vías respiratorias altas, traumas y laceraciones. Debido a las características anatómicas y fisiológicas típicas de esta especie, existe un gran riesgo en lo que se refiere a una anestesia general. El decúbito y su duración, eventualmente pueden causar desordenes cardio respiratorios y musculares, que pueden comprometer la recuperación de los ejemplares posterior a un procedimiento anestésico. El daño muscular, que puede generar una necrosis miopatía seria, producto de la presión e hipoxia generada en la masa muscular. Se ha observado que el decúbito dorsal es que más daño produce en contraste con el decúbito lateral izquierdo y el dercho, lo que se ha cuantificado en estudios midiendo la concentración sérica de las enzimas CK, LDH, AST. La prevención de este tipo de enfermedades se basa en la historia y manejo alimentario antes de ser sometidos a anestesia general.

Palabras clave: Miopatías, anestesia, caballo, enzimas séricas.

Universidad de Chile. Casilla 2 Correo 15 Santiago.

E-mail: agodoy@uchile 


\section{INTRODUCCIÓN}

Los Equinos Fina Sangre de Carrera (FSC) son atletas que se ven constantemente sometidos a grandes exigencias físicas, las que muchas veces traen como consecuencia alteraciones del sistema músculo esquelético. Muchas de estas alteraciones requieren de tratamiento quirúrgico y por lo tanto, de anestesia general, al igual de lo que sucede con las afecciones de las vías respiratorias altas y laceraciones producto de traumatismos múltiples.

En el equino, dadas las características anatómicas y fisiológicas propias de esta especie, son varios los riesgos que involucra la anestesia general, por lo que es fundamental que previo a ser sometido a ésta, se realice una completa revisión del paciente de manera tal que el protocolo anestésico que se use otorgue el máximo de seguridad, minimizando los riesgos pre y post anestesia y sin prolongar excesivamente el período de recuperación.

El decúbito al que es sometido un equino mediante anestesia general, dadas las características particulares de esta especie puede, eventualmente y dependiendo de su duración, provocar alteraciones cardiorrespiratorias y musculares que pueden comprometer la normal recuperación del paciente. Las anomalías respiratorias se deben fundamentalmente a las características anatómicas del pulmón del animal y al compromiso respiratorio del pulmón inferior. El daño muscular, que puede generar miopatías graves por necrosis, seria producto tanto de la presión ejercida por la gran masa muscular y esquelética, como también del compromiso hipóxico del tejido muscular.

Estas miopatías post-anestésicas, además de generar dolor y sufrimiento al animal, pueden retrasar su regreso al ejercicio, producir daño permanente e incluso en situaciones extremas conducir a la muerte. Dependiendo tanto de la duración del procedimiento quirúrgico como de la postura que el animal deba adoptar durante éste proceso, se pueden ocasionar secuelas post anestésicas.

\section{Miopatía post-anestésica}

Uno de los riesgos que conlleva toda anestesia general es la presentación de miopatía post- anestésica, que incluso podría llevar a tener la necesidad de someter a eutanasia al ejemplar (Duke et al., 2006) o bien muerte peri-operatoria (Raisis, 2005). Esta patología ocurre en un 6\% de los casos, y tiene dos formas de presentación, una de ellas es la localizada y la otra es la generalizada (Reed et al., 2004).

La miopatía post-anestésica fue durante mucho tiempo una complicación relativamente común de la anestesia general en caballos. Para fines de la década de 1980, se hizo claro que la ocurrencia de miopatías estaba directamente relacionada a la hipotensión que ocurre durante la anestesia inhalatoria (por ejemplo, con halotano). Desde ese entonces, el monitoreo de la presión arterial y el tratamiento oportuno de la hipotensión han reducido drásticamente la incidencia de miopatía post-anestésica en caballos (Wagner, 2009).

La hipoventilación y la hipoxemia son de común ocurrencia en caballos anestesiados. El aumento de la masa corporal puede acrecentar el riesgo de sufrir miopatías y neuropatías debido al daño por compresión en la mesa de cirugía. A pesar de las medidas preventivas factibles de ser utilizadas, como por ejemplo el acolchonamiento adecuado de la mesa, las miopatías pueden acaecer por perfusión inadecuada de los tejidos periféricos (Bidwell et al., 2007). La hipotensión, el decúbito prolongado, un acolchonamiento inadecuado, excesiva masa corporal, hipoxemia y un mal posicionamiento han sido identificados como factores de riesgo significativos para el desarrollo de miopatía post-anestésica en caballos y ponies adultos (Lucas y Satué, 2002; Cornick-Seahorn, 2004; Doherty y Valverde, 2006; Wagner 2009). La presión de perfusión adecuada es aparentemente importante para superar los efectos deletéreos de la alta presión intra-compartimental en los miembros que están en contacto con la mesa de cirugía y la presión hidrostática de los músculos en los miembros que están en el aire. La miopatía post-anestésica se puede desarrollar en parte debido a la disminución global de la perfusión sanguínea muscular como consecuencia de los efectos combinados de la baja presión hidrostática y arterial. Esta enfermedad está asociada a la depresión cardiovascular inducida tanto por halotano como por isofluorano, y la aminoración asociada del flujo sanguíneo muscular (Raisis, 2005). La patología en cuestión, más que un daño estructural por ne- 
crosis o destrucción de la fibra muscular, es atribuible a un proceso inflamatorio con aumento de permeabilidad de las fibras, ya que a las 24 horas post anestesia existe una recuperación de los niveles séricos de creatin fosfoquinasa (CK) mientras que los de transaminasa oxaloacética (AST) se mantienen altos (Godoy, 1992). La miopatía post-anestésica está asociada con necrosis muscular causada directamente por isquemia, e indirectamente por injuria por reperfusión. La isquemia tiene dos componentes, siendo uno de ellos la baja presión de perfusión secundaria a la hipotensión per se, y el otro el aumento de la presión intra-compartimental (Raisis, 2005).

Con respecto a la posición en si del animal, se ha observado que, en términos de la magnitud de la hipoxemia e hipercapnia, la posición de decúbito dorsal (Figura 1) es más perjudicial que el decúbito lateral (Figura 2), y que el decúbito lateral izquierdo produce una menor alteración que el decúbito lateral derecho. Por otra parte, se ha determinado que los valores de tensión de oxígeno y dióxido de carbono sólo vuelven a ser normales una vez que el animal está nuevamente en pie. Por estas razones se recomienda mantener al animal anestesiado el tiempo mínimo buscando aquellos agentes anestésicos que impliquen el período de recuperación más corto posible, y de ser posible según el procedimiento quirúrgico, preferir la posición de decúbito lateral sobre el decúbito dorsal (Lemus, 1990).

El daño muscular producido se debe en parte a que los equinos no están adaptados anatómica ni fisiológicamente a períodos prolongados de decúbito. Estudios han demostrado que el equino

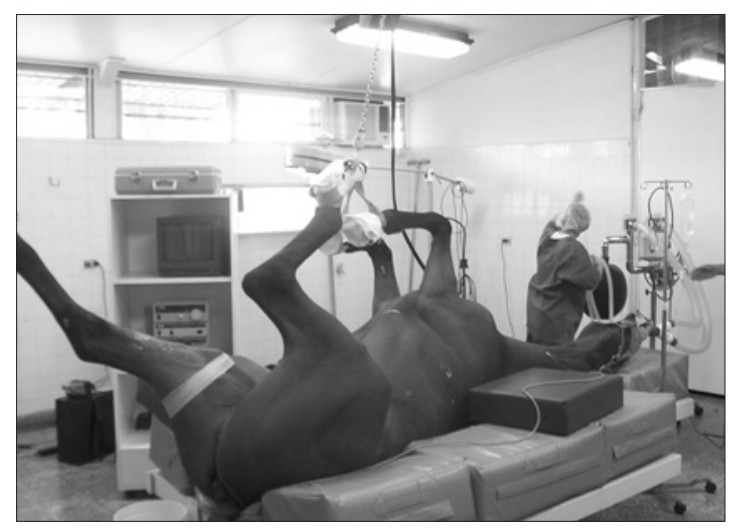

Figura 1. Equino en decubito dorsal. permanece sólo un $11 \%$ del día en posición de decúbito, siendo éste preferentemente esternal y repartido en 4 a 5 períodos de descanso sin adoptar voluntariamente la postura de decúbito dorsal. En términos generales las alteraciones respiratorias en el equino se deben fundamentalmente a dos factores; el gran tamaño de los pulmones, que dimensionalmente son más altos que anchos, y a la restricción de la ventilación del pulmón inferior, en el caso del decúbito lateral, que sería la porción inferior de ambos pulmones en caso del decúbito dorsal, abarcando un gran espacio, debido a la compresión mecánica producida por el peso de la pared torácica, de los elementos mediastinales, y en gran medida de las vísceras abdominales por la forma cónica del diafragma de esta especie (Godoy, 1992). Los grandes animales, como caballos y vacunos, son particularmente susceptibles a esta compresión del pulmón en decúbito dorsal debido al peso de las vísceras abdominales, que ejercen presión contra el diafragma, lo que produce la disminución de la ventilación en algunas aéreas pulmonares (Meyer et al., 2009).

Por efecto gravitacional, en el decúbito lateral el pulmón inferior recibe más sangre que el pulmón superior, sin embargo, el flujo sanguíneo en este último es mayor, lo cual se debe al aumento de la resistencia vascular pulmonar en el pulmón inferior, producida por la disminución mecánica de su volumen, originando a este nivel un encharcamiento importante de sangre. Este fenómeno se podría extrapolar al decúbito

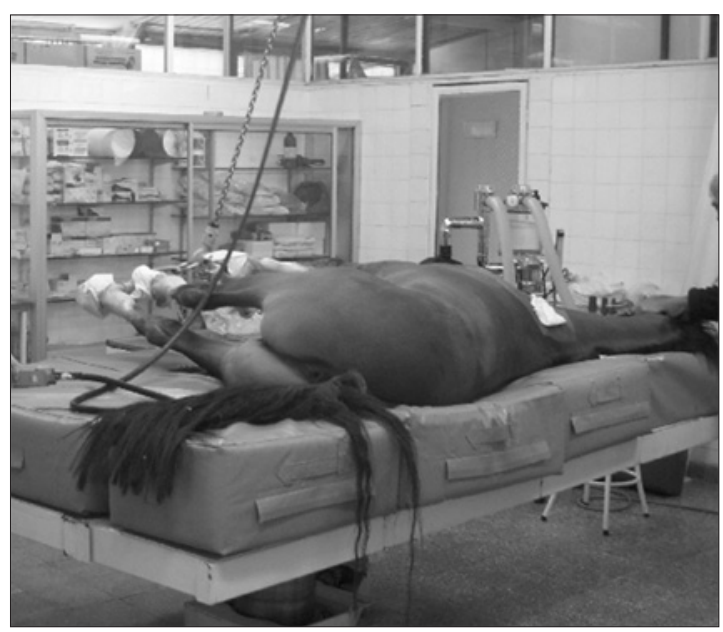

Figura 2. Equino en decubito lateral. 
dorsal, en el que la porción superior de ambos pulmones recibirían más sangre que la porción inferior, pero el flujo sanguíneo en este último sería mayor, con las implicancias antes descritas (Godoy, 1992).

Este desequilibrio entre ventilación y perfusión, descrito para el decúbito dorsal en grandes especies, puede llevar a oxigenación insuficiente de la sangre en las áreas comprimidas. Para llevar la sangre hacia zonas correctamente ventiladas del pulmón, ocurre vasoconstricción en las áreas hipóxicas. Si la compresión del pulmón se torna severa en animales adultos, el desequilibrio entre ventilación y perfusión antes descrito puede resultar en una mezcla de sangre sin oxigenar proveniente de las zonas hipóxicas del pulmón con la sangre correctamente oxigenada proveniente de las zonas correctamente ventiladas del pulmón en el lado izquierdo del corazón, disminuyendo la presión arterial de oxígeno (Meyer et al., 2009).

La restricción mecánica de la ventilación en el pulmón inferior, o porción superior en caso del decúbito dorsal, de un equino en posición de decúbito equivale a una reducción de un $48 \%$ de la capacidad funcional residual del volumen pulmonar. Este estado de hipoventilación del pulmón inferior determina que la sangre evacuada de éste, presente una presión parcial de oxígeno $\left(\mathrm{PO}_{2}\right)$ menor que la sangre evacuada del pulmón superior, o porción inferior (Godoy, 1992).

Esta gran desigualdad regional de perfusión y ventilación existentes entre los dos pulmones, o los dos sectores dentro del pulmón, en un equino en posición de decúbito permite explicar la diferencia que se observa a nivel alveolararterial de la presión parcial de oxígeno $\left(\mathrm{PO}_{2}\right)$, lo que a su vez es responsable de la abertura de cortocircuitos vasculares pulmonares de derecha a izquierda, tanto anatómicos como funcionales (Godoy, 1992).

Los signos típicos de cualquier enfermedad muscular incluyen músculos duros, compromiso sistémico, dificultad para mantenerse en pie (Godoy, 1992), atrofia, tumefacción focal, dolor a la palpación, en general músculos aumentados de tamaño, cambios de conducta, dificultad para aceptar ser ensillado, claudicación y paso acortado (Stashak, 2004), rendimiento pobre, intolerancia al ejercicio, debilidad, sudoración excesiva y frecuencia cardíaca y respiratorias excesivas asociadas al ejercicio (Alemán, 2005).

\section{Miopatía post-anestésica, forma localizada}

La forma localizada de la miopatia post anestésica es generalmente una consecuencia de la anestesia general, en combinación con una mesa quirúrgica extremadamente dura. Sin embargo, en algunos casos en que se emplea una colchoneta adecuada para amortiguar el efecto, el decúbito genera presión directa sobre las masas musculares o alteraciones de la perfusión por lo que el daño igual puede ocurrir debido al aumento en la presión dentro de los compartimentos osteofasciales, y una isquemia local. En este contexto, compartimento es definido como un músculo o grupo de músculos cercados por un sobre de fascia o periostio de baja elasticidad (Reed et al., 2004). Se han reportado durante la anestesia presiones intramusculares lo suficientemente fuertes para comprometer el flujo capilar e incluso afectar la transmisión neural (White y Suárez, 1986; Reed et al., 2004). La oclusión del flujo venoso produce una respuesta inflamatoria importante y una mayor reparación de tejido fibroso que la oclusión arterial (Reed et al., 2004).

La isquemia tisular es una causa importante del dolor. Cuando se interrumpe la perfusión de un tejido, éste se torna muy doloroso en pocos minutos. Cuanto mayor es la tasa metabólica del tejido, antes aparece el dolor. Una de las posibles causas de dolor durante la isquemia es la acumulación de grandes cantidades de ácido láctico en los tejidos como consecuencia del metabolismo anaeróbico durante la misma. También resulta posible que se formen en los tejidos otras sustancias químicas, como la bradicinina y las enzimas proteolíticas, a causa de la lesión celular y que sean éstas, y no el ácido láctico, las que estimulen las terminaciones nerviosas del dolor (Guyton y Hall, 2001).

Estudios recientes en el daño epitelial durante la isquemia sugieren que la mayoría de las células epiteliales sufren de muerte celular programada (apoptosis) durante la isquemia-reperfusión, más que necrosis, lo que permite la retención de componentes reutilizables de las células irreversiblemente dañadas (Reed et al., 2004). La lesión de reperfusión es iniciada durante la isquemia, cuando la enzima xantina deshidrogenasa es convertida a xantina oxidasa (Reed et al., 2004) y cuando su substrato, hipoxantina, se acumula simultáneamente debido al uso de ATP. Sin embargo, hay poca actividad de xantina oxidasa 
durante la isquemia, debido a que el oxígeno es requerido como un aceptor de electrones. Durante la reperfusión, la xantina oxidasa rápidamente degrada la hipoxantina en la presencia de oxígeno, produciendo el radical superóxido como un producto de esta reacción. El radical superóxido contribuye al daño tisular oxidativo y, más importante, activa las quimioquinas que atraen a los neutrófilos, lo que perpetúa el este daño (Reed et al., 2004).

Los radicales libres (por ejemplo superóxido e hidroxilo) y los elementos reactivos del oxígeno (por ejemplo, peróxido de hidrógeno) son metabolitos tóxicos del oxígeno generados durante el metabolismo celular aeróbico. Si es que estas moléculas son producidas en niveles que sobrepasan el mecanismo normal de defensa, son capaces de iniciar la peroxidación lipídica, llevando a la producción de radicales libres centrados en oxígeno y carbono, que pueden contribuir al daño tisular. Los radicales libres han sido implicados en muchos procesos patológicos. $\mathrm{Su}$ rol en la injuria por reperfusión ha sido estudiado en diversos órganos como el corazón, cerebro, intestino delgado y piel. El músculo esquelético puede encontrarse sujeto a isquemia, seguido por reperfusión durante varias circunstancias, incluyendo injuria térmica, trauma o preparación de colgajos musculares en cirugía reconstructiva (Reed et al., 2004).

Los radicales libres producidos después de la isquemia, y consecuente reperfusión, generan daño tisular, con aumento de la permeabilidad vascular, muerte celular y necrosis de tejidos. Todo esto genera anormalidades histológicas importantes (Doherty y Valverde, 2006).

La lesión isquémica es, al parecer, el factor iniciador de la miopatía post anestésica. El aumento en la presión muscular en el caballo en decúbito es tal que la perfusión muscular es disminuida hasta llegar al daño capilar. Esta baja de perfusión lleva a aumento en la permeabilidad de las paredes capilares, con la consiguiente formación de edema, el que perpetúa el aumento de la presión intracompartimental. La reperfusión del músculo isquémico está caracterizada por la disminución de la utilización de oxígeno, disminución del flujo sanguíneo y aumento en el tono vascular. El compromiso vascular también pareciera ser la causa de las miopatías de los miembros que no están en contacto con la mesa de cirugía, y que por lo tanto, no sufren de au- mento de presión intracompartimental (Doherty y Valverde, 2006).

Tanto un aumento en la concentración de lactato en la sangre que drena de los músculos comprometidos, como una hiperemia post-isquémica significativa en los músculos comprometidos y aumentos en la concentración plasmática de tromboxano y prostaglandina E2 han sido reportados en caballos anestesiados (Serteyn et al., 1990; Reed et al., 2004). Las prostaglandinas se generan en la membrana de la mayoría de las células nucleadas, la PGE2 relaja el músculo liso y genera vasodilatación, y junto con el tromboxano A2 estimulan la agregación plaquetaria y la liberación de mediadores plaquetarios como serotonina. El tromboxano (TXA2) hace que se contraiga el músculo liso y provoca vasoconstricción (Tizard, 1998). Es más probable que el daño producido sea resultado de la reperfusión de los músculos isquémicos, más que de la isquemia misma. La reperfusión origina oxidantes fuertes, que probablemente inician la peroxidación de la membrana lipídica y el daño muscular (Serteyn et al., 1990; Reed et al., 2004).

Los factores de riesgo asociados con miositis post-anestésica incluyen el peso y estado físico del caballo, posicionamiento, colchoneta de la mesa, duración de la anestesia, presión sanguínea (Duke et al., 2006), tipo de droga anestésica usada (Reed et al., 2004; Bidwell et al., 2007; Grint et al., 2007), perfusión muscular (Smith et al., 1996) y aplicación prolongada de un torniquete (Goodrich y McIlwraith, 2009).

La anestesia usando Halotano suscita una disminución del flujo de sangre al músculo $(0,40$ $\mathrm{ml} / \mathrm{min} / 100 \mathrm{~g}$ de músculo), en comparación con la presión observada en el músculo sin aneste$\operatorname{siar}(1,20 \mathrm{ml} / \mathrm{min} / 100 \mathrm{~g}$ de músculo) (Reed et al., 2004). A su vez, el Halotano provoca desequilibrios electrolíticos en mamíferos los que influyen en la función muscular y pueden asociarse con daño al tejido muscular (Mitchell et al., 1999). Existe una predisposición a la isquemia a través de ciertas enfermedades o estados patológicos coligados con hipotensión y/o disminución de la perfusión muscular (Reed et al., 2004). La perfusión muscular también puede verse disminuida si es que existe vasoconstricción periférica, cuya causa podría atribuirse entre otros motivos, a la liberación de catecolaminas como consecuencia de la excitación previa a la anestesia (Grint et al., 2007). 
Generalmente, se observan signos clínicos en el miembro que está más abajo (comprimido) en decúbito lateral. En los miembros anteriores, el músculo tríceps (Smith et al., 1996; Lucas y Satué; 2002; Reed et al., 2004; Stashak, 2004), deltoides (Lucas y Satué; 2002; Reed et al., 2004), y ocasionalmente el braquiocefálico y pectoral craneal son los que generalmente se ven afectados. En los miembros posteriores, generalmente se ven perjudicados los músculos bíceps, vasto femoral (Reed et al., 2004) extensores, y longísimo en decúbito lateral (Lucas y Satué; 2002). Los músculos del flanco y el macetero también suelen verse influidos. Cuando un caballo es anestesiado en decúbito dorsal, los músculos glúteos y ocasionalmente longíssimus dorsi tienden a observarse dañados. Circunstancialmente el miembro que está en alto puede resultar expuesto si su recirculación se ve comprometida (Reed et al., 2004). El drenaje venoso desigual entre ambos elementos puede ser un factor que contribuya con la presentación de miositis post-anestésica, a lo menos cuando se compara con el segmento que está en alto (Reed et al., 2004).

La condición descrita con anterioridad genera dolor severo y claudicación y, aunque originalmente era considerada como una neuropatía, en la actualidad se acepta en forma primordial como una miopatía eventualmente con componentes neurológicos (Smith et al., 1996). Los signos clínicos de la miositis post-anestésica en general se manifiestan durante la recuperación inicial, aunque en forma ocasional pueden tardar aproximadamente una hora en aparecer. En un comienzo puede sospecharse de la condición al ser demasiado larga la recuperación o al hacer el caballo intentos repetidos por pararse sin éxito. Signos clínicos de daño de un paquete muscular, un nervio periférico, o ambos, son observables. El grupo muscular afectado tiende a aumentar ostensiblemente su temperatura pudiendo incluso ser percibida al tacto, además de sufrir un aumento en su volumen observable a simple vista. El caballo resiente la palpación y generalmente no quiere apoyar la extremidad o no soporta carga. Se pueden evidenciar signos de dolor severo, con sudor. Generalmente, cuando los músculos de los miembros anteriores son los afectados, el caballo permanece de pie como si el nervio radial estuviera paralizado, pero puede hacer uso de sus músculos extensores. En la ocasión en que los miembros posteriores son los afectados, puede ocurrir flexión del nudo; si es el cuadriceps femoral el que se ve afectado, la babilla y tarso pueden colapsar impidiendo que el caballo se ponga de pie, especialmente si ambos miembros posteriores están dañados. Los signos pueden persistir por varios días, incluso en casos no tan complicados (Lucas y Satué; 2002; Reed et al., 2004; Stashak, 2004).

Dentro de los grupos musculares afectados, la miopatía del cuádriceps se reconoce clínicamente por una claudicación de el o los miembros afectados, que son incapaces de soportar peso, iniciada desde la articulación femoro tibio rotuliana hacia arriba (de Oliveira et al., 2008) y puede existir dificultad para trabar la patela. Esta patología se puede prevenir flectando la articulación femorotibiorotuliana que no está siendo operada durante la cirugía (Goodrich y McIlwraith, 2009). En casos de miopatía del músculo tríceps, cuando el caballo está en estación, el codo tiene una clásica apariencia de estar "caído", y el caballo no puede cargar peso en la extremidad afectada. Puede existir una apariencia similar cuando hay neuropatía o fractura de la ulna (Wagner, 2009).

En algunos caballos, las únicas anormalidades observables son placas dolorosas aisladas y aumentadas de tamaño, y se pueden encontrar junto con disfunción de los miembros anteriores o posteriores. Estos aumentos de tamaño generalmente ocurren en las zonas de contacto con la mesa de cirugía (caderas, tórax o área facial) y pueden ser causados, como se indicara con anterioridad por una colchoneta inadecuada, trauma, o por el peso del paciente (Lindsay et al., 1989, Reed et al., 2004).

La alta presión intracompartimental también puede alterar la transmisión nerviosa, algo que se observa con frecuencia en el síndrome compartimental en las personas y en los perros (Stashak, 2004). Los nervios superficiales, como el facial y el radial, están sometidos a daño por presión durante el decúbito. Se le debería prestar especial atención a proteger y acolchar estos nervios para evitar este daño (Wagner, 2009). En consecuencia, tanto las anormalidades de los músculos como las de los nervios pueden coexistir, haciendo difícil la diferenciación clínica. Con las neuropatías se pueden observar signos clínicos similares a las miopatías localizadas excepto que no serán evidentes las anormalidades en los músculos. Los niveles séricos de las enzimas muscu- 
lares también pueden ser utilizados para ayudar a la diferenciación entre una neuropatía y una miopatía localizada en el paciente postanestésico (Stashak, 2004).

Las neuropatías son injurias en el sistema nervioso, relacionadas con la anestesia. Generalmente involucran nervios periféricos aunque pueden afectar la médula espinal. Muchos de los factores que influyen en el desarrollo de miopatía post anestésica también son importantes en el desarrollo de las neuropatías, como por ejemplo, gran masa corporal, duración del decúbito y acolchonamiento de la mesa quirúrgica. Los signos clínicos de estas afecciones dependen de la severidad y localización del daño nervioso, y generalmente se notan cuando el caballo trata de ponerse de pie. Esta condición generalmente ocurre en el nervio del miembro que está más cercano al colchón de la mesa quirúrgica (Doherty y Valverde, 2006).

El nervio radial tiene más posibilidades de ser afectado cuando el caballo está en decúbito lateral (Wagner, 2009). Cuando hay daño en este nervio, los signos clínicos varían con la severidad de la injuria, y son evidentes cuando el caballo intenta ponerse de pie, aunque pueden desarrollarse horas después de la cirugía. El caballo es incapaz de soportar peso en el miembro afectado, o extenderlo, y el codo de este miembro se ve caído. Generalmente, no hay dolor en el área afectada, excepto que haya una miopatía concomitante del tríceps. En el caso de que ambas patologías estén presentes, es difícil diferenciarlas. Usualmente cuando sólo hay daño del nervio radial no hay inflamación ni dolor. De manera de evitar la aparición de esta patología, el miembro que está más cerca de la mesa quirúrgica debería ser tirado hacia delante de manera de liberar el nervio y el músculo tríceps de debajo de la caja torácica (Doherty y Valverde, 2006). Un problema común que produce daño en este nervio es el acolchonamiento insuficiente, o colchonetas que no soportan completamente la zona del hombro y codo, sino que terminan en algún lugar entremedio de estas estructuras, ejerciendo con sus bordes una presión indebida sobre el área donde está el nervio. Existe una posición característica con el hombro caído, por lo que se debe diferenciar, mediante radiografías y examen físico, esta patología de una fractura de olecranon o miopatía del tríceps (Wagner, 2009).

El daño al nervio femoral probablemente es resultado de la sobreextensión de los miembros pélvicos cuando el caballo se encuentra en decúbito dorsal. Los signos clínicos pueden verse en uno o ambos miembros pélvicos, e incluyen la incapacidad de extender la articulación femorotibio-rotuliana, con una flexión evidente del tarso y generalmente no existe inflamación. Si ambos miembros están afectados, el caballo es incapaz de ponerse de pie. La recuperación de la anestesia es difícil en estos casos, y la recuperación de los casos suaves se completa en pocos días (Doherty y Valverde, 2006).

Puede existir daño del nervio facial cuando la hebilla de la jáquema hace presión sobre este, con el caballo en decúbito lateral (Doherty y Valverde, 2006), o cuando se realizan cirugías que involucran la cabeza o el ojo, cuando las manipulaciones quirúrgicas someten a la cabeza a presión o peso excesivos (Wagner, 2009). Se observa parálisis motora de los músculos faciales, siendo la caída del labio el signo clínico más común (Doherty y Valverde, 2006) y parálisis parcial de un lado de la cara (Wagner, 2009). Generalmente la condición resuelve en pocos días, siendo pocos los casos en que se vuelve permanente (Doherty y Valverde, 2006).

El tratamiento de las neuropatías generalmente es de soporte, se debe ayudar al caballo a ponerse de pie, y en caso de que esto no sea posible, se debe asegurar un acolchonamiento adecuado y cuidados de sostén, los que involucran mantener una correcta hidratación (Doherty y Valverde, 2006), con tratamiento antiinflamatorio y analgésico (Doherty y Valverde, 2006; Wagner, 2009). Se recomienda el uso de DMSO (dimetil sulfóxido) y tratamientos locales con ultrasonido y masajes (Doherty y Valverde, 2006).

La actividad de las enzimas CK y AST aumenta en los individuos dañados y se puede observar mioglobinuria con dolencia muscular significativa (Reed et al., 2004).

\section{Miopatía post-anestésica forma generalizada}

La forma generalizada de la miopatia post anestésica puede ser causada por una condición similar a la hipertermia maligna de los humanos y los suinos. Los signos pueden aparecer durante la recuperación, con muchos grupos musculares afectados, independientes de la posición del caballo en la mesa de cirugía (Reed et al., 
2004). Los signos clínicos incluyen: inflamación muscular generalizada, orina color café oscuro, sudoración profusa, manoteos, temblores, incapacidad para ponerse de pie, aumento sérico de las enzimas musculares. Se pueden observar fasiculaciones musculares en la recuperación anestésica temprana (Grint et al., 2007).

Las causas de esta forma aún no han podido ser precisadas con exactitud, pero se ha sugerido que es el resultado tanto como producto de la isquemia local, la que se torna más generalizada aún como consecuencia de la hipotensión, como de la sensibilidad de las células musculares a las drogas anestésica o a los relajantes musculares depolarizantes, o a una combinación de los factores mencionados precedentemente (Reed et al., 2004).

No es del todo conocido ni ha podido ser establecido con exactitud aún si un caballo con miopatías hereditarias es más o menos susceptible de sufrir una miopatía generalizada post-anestésica o un cuadro de hipertermia maligna, por lo que constantemente es adecuado, previo a una anestesia general, ejecutar una buena anamnesis en la que se incluyan estos datos, ya que el potencial de posibilidades de que se presente la enfermedad, existe (Reed et $a l, 2004)$. Se ha documentado manifestaciones de miopatías post-anestésicas en caballos que sufren de esta enfermedad por acumulación de polisacáridos (Colgan et al., 2006).

La forma generalizada puede ocurrir en cualquier momento durante el transcurso de la aplicación de la anestesia y como se insinuara anteriormente, la temperatura, frecuencia cardíaca y respiratoria aumentan, y los músculos pueden fascicularse y contraerse, el caballo se resiste a la ventilación asistida e incluso su muerte es posible (Reed et al., 2004).

Casos post-operatorios también pueden ocurrir, independientes de la duración de la anestesia y del posicionamiento. Se observan signos de dolor extremo (Reed et al., 2004) y generalmente el animal es incapaz de levantarse o mantenerse erguido (Lucas y Satué, 2002; Reed et al., 2004). Los músculos pueden estar rígidos y hay una sudoración profusa. Es factible de que existan signos de cólico, mioglobinuria y desequilibrio hídrico (Reed et al., 2004); y/o manifestaciones de falla renal aguda tanto en la forma localizada como en la generalizada (Lucas y Satué, 2002).

La recuperación de las formas agudas conduce a fibrosis y a atrofia muscular. Las más afectadas son las fibras de contracción rápida tipo II (Lucas y Satué, 2002).

Las pruebas diagnósticas para determinar la presencia de enfermedad muscular incluyen entre otras el examen físico, la termografía, la ecografía, la medición de la concentración sérica/ plasmática de las enzimas y la biopsia muscular (Stashak, 2004).

\section{Diagnóstico de las miopatías post-anestésicas}

En ambas formas de presentación, las concentraciones plasmáticas de CK, AST y lactato deshidrogenasa (LDH) se encuentran aumentadas (Lucas y Satué, 2002; Reed et al., 2004). Esto permite diferenciar los animales con daño muscular, de aquellos que sufren de condiciones similares a la parestesia (posiblemente causada por una baja irrigación a los terminales nerviosos sensitivos). Puede existir un desequilibrio hídrico o electrolítico. Se ha documentado presencia de hipocalcemia, hipomagnesemia, hiperfosfatemia, acidosis metabólica, hiperkalemia e hiperglicemia, y en el caso de estar presentes, debieran ser tratadas. Puede ocurrir mioglobinuria, comprometiendo la función renal. En caso de hacer una biopsia, el examen histológico es concordante con rabdomiolisis (Reed et al, 2004). La molestia muscular puede ser secundaria a otra enfermedad, obligando a que un examen físico completo sea fundamental para descartar otras anormalidades (Stashak, 2004).

Las enzimas que son más útiles en la evaluación del sistema muscular equino son las CK, AST y LDH. Un cambio en la actividad plasmática de una enzima puede ocurrir por variados motivos, incluyendo alteraciones en la permeabilidad de la membrana de la célula que contiene las enzimas, necrosis celular, clearance o eliminación defectuosa de la enzima, y aumento o defectos de síntesis. La disminución de los niveles plasmáticos de una enzima suelen no ser clínicamente significativos (Reed et al., 2004). La actividad enzimática se utiliza principalmente para coadyuvar en el diagnostico de la enfermedad muscular generalizada en los caballos, y debe interpretarse según la situación clínica (Stashak, 2004).

Como se mencionó anteriormente, la enzima CK es la enzima responsable de degradar la 
creatinina fosfato en creatinina y fosfato (Reed et al., 2004), catalizando la formación del ATP necesario para la contracción muscular (Rudolph, 2003), siendo esta reacción es la única fuente de energía para el músculo en el inicio de la actividad. En el caballo, esta enzima se encuentra básicamente en el músculo esquelético, el miocardio y el cerebro. La vida media plasmática de la CK es corta (108 minutos, $123 \pm 28$ minutos con un clearance plasmático de $0,36 \pm 0,1 \mathrm{ml} /$ $\mathrm{kg} / \mathrm{min}$ ) (Reed et al., 2004). Con una lesión muscular aguda, la actividad de la CK alcanza su nivel máximo a partir de las 2 y hasta las 6 horas (Stashak, 2004) y vuelve a la normalidad a los 3 a 4 días (Leadon, 2006). Los niveles mantenidos elevados de CK en el suero, indican que la necrosis muscular permanece activa. En cambio, en las lesiones musculares crónicas, o cuando la mionecrosis deja de ser activa, el nivel circulante de CK puede disminuir hasta su nivel normal o permanecer sólo ligeramente elevado. (Rudolph, 2003). El rango de referencia para esta enzima es de 60-330 UI/litro (Doherty y Valverde, 2006).

Tal como se explicó anteriormente, la enzima AST se encuentra principalmente en el músculo esquelético, el hígado y el corazón, a pesar de que existe baja actividad en otros tejidos, por lo que la AST no es específica de un solo tipo de estructura muscular (Rudolph, 2003; Reed et al., 2004). La vida media plasmática de la AST en el caballo es de 7 a 10 días (Reed et al., 2004; Stashak, 2004), por lo que permanece elevada hasta una semana después de una lesión puntual, cuando se producen lesiones repetidas, los niveles tienden a permanecer altos (Rudolph, 2003). La función de AST alcanza su máxima expresión a las 24 horas (Stashak, 2004). El nivel sérico de AST es útil para diagnosticar enfermedades musculares siempre y cuando se emplee conjuntamente con los niveles de CK o de las isoenzimas LDH. Mientras que el nivel sérico de la enzima $\mathrm{CK}$ se incrementa rápidamente en las miopatías, los niveles de LDH y de AST lo hacen de forma lenta (Rudolph, 2003).

La diferencia en las actividades enzimáticas pueden ser utilizadas para evaluar el estadio (agudo, crónico, en recuperación) del compromiso muscular en los caballos con rabdomiólisis (Stashak, 2004).

Las pruebas diagnósticas para precisar la existencia de alguna enfermedad muscular incluye el examen físico, la termografía, la ecografía, la medición de la concentración sérica/plasmáti- ca de las enzimas y la biopsia muscular (Stashak, 2004).

En el examen físico es necesario contemplar la inspección en busca de asimetría y la palpación digital directa de los músculos sospechosos a fin de detectar dolor, calor y consistencia, y comparar las posibles diferencias de estos con los músculos del lado opuesto. Debe realizarse un examen completo de la claudicación para determinar si el problema muscular es una lesión primaria o puede ser secundario a otros trastornos del aparato músculo esquelético (Stashak, 2004).

En ciertas ocasiones, la ecografía resulta beneficiosa para el diagnóstico de lesiones musculares en el caballo (Stashak, 2004). El ultrasonido puede proveer de un elemento evaluador adicional para medir la extensión del daño de la musculatura y determinar la recuperación del caballo, a la vez que sirve para hacer una estimación del pronóstico del caballo. En una ecografía, se piensa que los ecos son generados por los fascículos musculares y el perimisio, mientras que el tejido muscular propiamente tal es anecoico (Smith et al., 1996). La solución de continuidad muscular no siempre es visible, pero por lo general hay un hematoma/seroso en la vecindad de la lesión del tejido, sugiriendo daño importante en el mismo (Stashak, 2004).

El examen microscópico del tejido muscular (biopsia) se utiliza cada vez con mayor frecuencia para el diagnóstico de enfermedades del mismo. Las biopsias musculares están indicadas en los caballos que tienen episodios repetidos de rabdomiólisis y que no responden al tratamiento estándar (Stashak, 2004). Se puede utilizar dos técnicas de biopsia siendo la primera de ellas la quirúrgica abierta o por aguja percutánea (Reed et al., 2004; Stashak, 2004), existiendo además la biopsia muscular que permite examinar las propiedades morfológicas, bioquímicas y fisiológicas de las miofibras. Los puntos indicados para la obtención de muestras para biopsias musculares, en caso de miopatía generalizada, son el semimembranoso, el bíceps femoral y el glúteo medio, siendo las técnicas adaptables a cualquier músculo en caso en miopatía localizada (Reed et al., 2004). La biopsia muscular confirma la patología, caracterizada por hinchazón mitocondrial, disrupción de las crestas y división fibrilar, cambios comunes con las rabdomiolisis agudas (Lucas y Satué, 2002).

La termografía es una herramienta diagnósti- 
ca empleada en la detección de la temperatura de la superficie corporal. A pesar de detentar ciertas limitaciones, puede ser usada como una herramienta diagnóstica útil, la que complementa la palpación digital y ayuda a la confirmación de lesiones sospechosas en tejidos blandos/musculares (Stashak, 2004). El Médico Veterinario puede hacer uso de esta técnica no invasiva de manera de localizar los cambios en la temperatura de la piel que se deben principalmente a variaciones en el flujo sanguíneo periférico. La inflamación, la atrofia, la neoplasia y las lesiones neurológicas pueden ser causantes de las alteraciones en el flujo sanguíneo local (Reed et al., 2004).

La Electromiografía (EMG) se refiere a los cambios en el potencial eléctrico asociado con la unidad motora (Reed et al., 2004; Stashak, 2004). El status eléctrico de la membrana muscular depende de la integridad de la unidad motora completa (Reed et al., 2004). Las agujas de los electrodos se introducen en el músculo y son utilizadas para captar los potenciales de acción, los que estarán ausentes o serán anormales ante la presencia de una enfermedad muscular. La técnica descrita es útil para indicar lesión muscular y es empleada principalmente para identificar miopatías de origen neurogénico (Stashak, 2004).

En el urianálisis de un caballo con daño muscular es posible detectar mioglobinuria. La mioglobina resulta esencial para el transporte de oxigeno hacia y entre las células musculares. La mayoría de los mamíferos contienen $1 \mathrm{mg}$ de mioglobina por gramo de tejido muscular fresco, y se insinúa que debieran destruirse al menos 200 gramos de tejido muscular, de forma aguda, para que los niveles séricos de mioglobina aumenten lo bastante que permita que ésta sea detectada en la orina. La pigmenturia puede ser causada tanto por aumento de mioglobina, de hemoglobina, o de sangre entera, pero los niveles de esta deben ser lo suficientemente elevados para que el cambio de color sea visible. No se puede distinguir la mioglobinuria solo por el color, es por ello que existen variadas pruebas de laboratorio que distinguen el pigmento que causa la pigmenturia (Reed et al., 2004).

\section{Tratamiento de las miopatías post-anestésicas}

El objetivo final del tratamiento de las miopatías post-anestésicas es aliviar el dolor, prevenir que el daño se perpetúe, corregir los desequilibrios hídricos y electrolíticos, y mantener la función renal (Lucas y Satué, 2002; Reed et al., 2004). Este tratamiento recomendado generalmente es similar al de la miositis por exceso de ejercicio. En caso de existir acidosis metabólica, el caballo podría requerir de la aplicación de bicarbonato de sodio por vía endovenosa. Al aparecer los signos mientras el paciente está sometido a anestesia general, ésta debiera ser interrumpida y requeriría cambiársele el agente anestésico por uno alternativo endovenoso; al aparecer señales de hipertermia se hace necesario enfriar el caballo lo antes posible (Reed et al., 2004), y administrar fluido terapia. Los analgésicos usados incluyen antiinflamatorios no esteroidales (AINES) (Reed et al., 2004; Doherty y Valverde, 2006; Wagner, 2009) y opioides (Reed et al., 2004). Otro tratamiento recomendado es el uso de DMSO (dimetil sulfóxido) (Doherty y Valverde, 2006). Otra posibilidad, de manera de reducir el espasmo muscular, es el uso de Diazepam y Guafenesina, pero se debe que tener cuidado debido a que puede presentarse ataxia, lo que dificultaría aún más el proceso de puesta en pié del caballo (Reed et al., 2004). El paciente puede requerir sedación cuando esté lúcido, con la finalidad de disminuir la ansiedad generada por el dolor (Grint et al., 2007). Los $\alpha$-agonistas (xilacina, detomidina, romifidina) son capaces de proveer una buena sedación y analgesia, pero podrían exacerbar la ataxia, promover la sudoración, la hipoinsulinemia e hiperglicemia, y potenciar la producción de orina, lo que conlleva a una agudización de la pérdida de líquido. El efecto vasoconstrictor de todos estos agentes, sumados al impacto que tienen sobre el gasto cardiaco puede comprometer aún más la perfusión de los tejidos. Es posible que su uso en los casos más agresivos sea necesario (Reed et al., 2004). Es posible proveer una buena sedación, con el empleo de acepromacina (Reed et al., 2004; Wagner, 2009), en conjunto con opiodes, lográndose de esta forma un mínimo de efecto de ataxia, y su impacto vasodilatador podría ayudar a mejorar la perfusión de los tejidos, asumiendo que el volumen sanguíneo es mantenido, previniendo la hipovolemia (Reed et al., 2004).

Se puede aplicar medidas para disminuir la temperatura de las zonas afectadas y que tienen alto flujo sanguíneo en el cuerpo, como son el surco yugular, entre los muslos y en las 
axilas (Klein, 1990). Si el caballo permanece en decúbito, resulta fundamental disponer de una superficie blanda y modificar la posición del animal para evitar compresiones continuas (Lucas y Satué, 2002; Doherty y Valverde, 2006).

Algunos caballos necesitan ayuda para ponerse de pie. En casos en que los signos clínicos no son severos, los caballos se pueden beneficiar de ejercicio ligero (caminatas), empezando al día siguiente de la cirugía. Se recomiendan los tratamientos locales, como ultrasonido y masajes (Doherty y Valverde, 2006).

\section{Prevención de las miopatías post- anestésicas}

No se conocen medidas preventivas específicas para evitar estas complicaciones post anestésicas, sin embargo, la determinación de niveles séricos de enzimas tales como AST y CK, junto a la de otros parámetros del individuo, previo a la anestesia, resultan de gran utilidad para la identificación de aquellos ejemplares de alto riesgo (Godoy, 1992). Asimismo, es importante señalar en forma reiterativa, dado a la trascendencia que en si reviste la materia, la necesidad de mantener un posicionamiento adecuado en la mesa quirúrgica (Lucas y Satué, 2002; Reed et al., 2004; Doherty y Valverde, 2006), a fin de prevenir la restricción del drenaje venoso o de la irrigación arterial (Reed et al., 2004). En una dirección similar y relacionada con la materia resulta de interés redundar acerca de lo aconsejable de mantener un acolchonamiento adecuado (Reed et al., 2004; Doherty y Valverde, 2006; Tranquilli et al., 2007), se ha concluido y reportado que las camas de espuma son de inferior calidad que los colchones de aire o de agua (Reed et al., 2004).

El posicionamiento es muy importante, y existe muchas recomendaciones al respecto, tales como la que indica que se debiera elevar el miembro que está más arriba para reducir la presión el tríceps del que está más abajo, llevándolo hacia delante (Reed et al., 2004; Doherty y Valverde, 2006). En el decúbito lateral, debiera mantenerse los miembros posteriores paralelos o más arriba, con respecto a la mesa, y suficientemente separados para promover el drenaje venoso. En el decúbito dorsal, en vez de dejar que los miembros posteriores se posicionen de forma pasiva, se les debe proveer de soporte en ligera extensión. Las miopatías en los músculos abduc- tores de los miembros posteriores no siempre se desarrollan con posicionamiento pasivo. Se debiera evitar arrastrar los miembros hacia atrás, $\mathrm{y}$ si resulta necesario hacerlo, se debería reducir la cirugía al tiempo mínimo evitando por este medio las miopatías del músculo cuádriceps. Cuando el cirujano necesita acceder a la cara medial del carpo y radio distal, la posición horizontal flectada hacia delante, con el miembro que está más arriba, descomprimido, paralelo al piso, totalmente flectado al carpo, y gentilmente empujado hacia adelante, es más aconsejable (Reed et al., 2004). Cuando el caballo está en decúbito dorsal, se debería posicionar cuadrado sobre su dorso. Se debería evitar la flexión o sobreextensión de los miembros. Los miembros que no están apoyados en la mesa de cirugía se deben posicionar paralelos a dicha mesa, y no se debe colocar colchonetas sobre los miembros dependientes de manera de apoyar los miembros que están más arriba, ya que esto afecta el drenaje venoso de los miembros dependientes (Doherty y Valverde, 2006).

Excluir de la ración los granos durante 48 a 72 horas previo a la cirugía puede resultar beneficioso, ya que el consumo elevado de ello se ha asociado a la presentación de miopatía post-anestésica (Lucas y Satué, 2002; Reed et al., 2004). Los caballos pesados, mestizos y los más atléticos pueden tener más riesgo de sufrir miositis post anestésica, particularmente si es que tienen lesiones inducidas por el ejercicio (Reed et al., 2004).

Es importante mantener el plano anestésico lo más bajo posible, controlando la presión arterial y evitando la hipovolemia (Lucas y Satué; 2002). Durante el desarrollo de la cirugía es conveniente mantener la presión arterial media sobre los 70 mmHg (Cornick-Seahorn, 2004; Duke et al., 2006; Tranquilli et al., 2007) u $80 \mathrm{mmHg}$, minimizar el uso de Halotano, y usar fluidoterapia intraoperatoria (Reed et al., 2004), además se debe considerar la posibilidad de reducir la duración de la anestesia lo más posible, mantener al paciente bien oxigenado y sostener un plano mínimo de anestesia quirúrgica con mínima depresión cardiorrespiratoria (Godoy, 1992). En situaciones en que la presión sanguínea disminuye del nivel mencionado anteriormente, es aconsejable administrar agentes inotrópicos (CornickSeahorn, 2004).

Los caballos anestesiados generalmente de- 
sarrollan hipoxemia debido a la alteración de la ventilación y la perfusión, y al "shunt" intrapulmonar de derecha a izquierda. La hipoxemia se desarrolla con mayor frecuencia en pacientes mantenidos en decúbito dorsal, y puede ser evitada mediante la temprana aplicación de ventilación intermitente con presión positiva (CornickSeahorn, 2004).

Se ha evaluado la influencia de varios simpaticomimeticos en el tráfico sanguíneo muscular de caballos anestesiados con halotano, en decúbito lateral. La Dopamina y la Dobutamina incrementan la circulación del flujo sanguíneo muscular del tríceps que no queda apoyado directamente sobre la mesa quirúrgica, pero sólo la Dobutamina mejora la perfusión del músculo del miembro que ha quedado comprimido debido al apoyo directo sobre la mesa de operaciones. A su vez, se ha comprobado que la Dopamina es de gran utilidad para el aumento del gasto cardíaco en caballos anestesiados (Duke et al., 2006). La Dobutamina incrementa la presión arterial, el gasto cardiaco, y el flujo sanguíneo intramuscular, siendo este último relevante en la prevención de la miopatía post-anestésica. La Efedrina provee una intensificación sostenida en la presión arterial y el gasto cardíaco en caballos anestesiados con Halotano (Cornick-Seahorn, 2004).

En animales de alto riesgo, se ha sugerido la administración profiláctica de dantrolene (1-2 $\mathrm{mg} / \mathrm{kg}$ oral, 1-2 h previa a la inducción anestésica) (Lucas y Satué; 2002).

\section{Pronóstico de las miopatías post-anestésicas}

El pronóstico depende parcialmente de la amplitud del deterioro muscular, del tratamiento instaurado, y del temperamento del individuo. Generalmente los caballos se recuperan completamente, en forma especial si es que la condición se localiza solamente en un grupo muscular. Ocasionalmente, el paciente puede quedar con atrofia muscular posterior a la cirugía y fibrosis, y el deceso del mismo puede ocurrir, con áreas masivas de mionecrosis isquémica y degeneración intrafascicular de la fibra nerviosa, como lo demuestran las lesiones halladas en las necropsias. Se podría poner en práctica la eutanasia como alternativa extrema y de orden netamente humanitario en caballos que permanecen postrados, ante una rabdomiolisis severa o un dolor in- manejable y/o fuera de control médico (Reed et al., 2004).

\section{Diagnóstico diferencial de las miopatías post- anestésicas}

La Hipertermia Maligna (HM) es una enfermedad infrecuente y de ocurrencia extraordinaria (Lucas y Satué, 2002; Doherty y Valverde, 2006) que afecta el sistema músculo-esquelético, de raíces fármaco-genética que puede conducir al deceso del paciente (Alemán, 2008). Esta enfermedad es hereditaria (Reed et al., 2004) y gatillada por la exposición del enfermo a anestésicos inhalatorios, tal como el Halotano, a los relajantes musculares depolarizantes como la succinilcolina, y al estrés (Aleman, 2005). Mutaciones en los genes R1 y R2 ocasionan disfunción de los canales de Calcio del retículo sarcoplásmico en las células del músculo esquelético, generando un paso excesivo de Calcio al mioplasma y un estado hipermetabólico caracterizado por calor intenso, hipercapnia, acidosis láctica y en ocasiones, deceso del paciente (Lucas y Satué; 2002; Reed et al., 2004; Grint et al., 2007; Aleman; 2008). En su formato clásico, la enfermedad está singularizada por la hipertermia, la rigidez, las contracciones musculares y el test de contractibilidad in vitro anormales, todo ello tras la administración de algunos agentes anestésico inhalatorios o relajantes musculares (Lucas y Satué; 2002). La HM en caballos se ha desencadenado por la sola aplicación del Halotano, o al haberlo suministrado en combinación con succinilcolina, cafeína o en conexión con la estimulación nerviosa (Reed et al., 2004; Alemán; 2008), con el estrés o con una actividad muscular superior al nivel de entrenamiento del animal (Lucas y Satué, 2002). En apariencia no existirían señales de predisposición de raza, de género o de edad en caballos (Aleman; 2008).

Los signos clínicos más comunes para la HM en caballares son de manifestación lenta (Grint et al., 2007) y estas señales incluyen hipertermia, que puede exceder $\operatorname{los} 43^{\circ} \mathrm{C}$, sudoración profusa, rigidez muscular, taquicardia, (Lucas y Satué, 2002; Reed et al., 2004; Aleman, 2005; Alemán, 2008) taquipnea (Grint et al., 2007), arritmia cardíaca, prolapso del tercer párpado, contracciones musculares, rabdomiolisis, mioglobinuria, y deceso con rigor mortis peragudo. El diagnóstico 
previa a la anestesia es muy engorroso y complejo (Alemán, 2008).

Lasanormalidadesclinicopatológicasincluyen hipercapnea, acidosis, aumento progresivo del volumen arterial de $\mathrm{CO}_{2}$, de la kalemia, del $\mathrm{Pi}$, hipertensión (Lucas y Satué, 2002; Grint et al., 2007), desórdenes electrolíticos, el aumento de CK sérica y la mioglobinuria. El incremento de la actividad de las enzimas musculares puede no ser observada en casos de muerte peraguda. No existen anormalidades histoquímicas específicas en la biopsia muscular de caballos afectados (Alemán, 2008). La evaluación diagnostica se realiza por la sintomatología en el transcurso de una cirugía. De hecho, el aumento progresivo de la temperatura corporal es bastante indicativo (Lucas y Satué; 2002).

El tratamiento para lo precedentemente expuesto consiste en suspender la anestesia, bajar la temperatura y usar relajantes musculares como el dantrolene (Lucas y Satué, 2002).

Se ha sugerido que existe una asociación entre la hipertermia maligna, la rabdomiolisis por ejercicio y la parálisis peródica hipercalémica (Lucas y Satué, 2002).

\section{CONCLUSIONES}

Los equinos fina sangre de carreras sufren en forma frecuente patologías, muchas de las cuales requieren tratamiento quirúrgico y por ende deben ser sometidos a anestesia general. Esta, además de los riesgos inherentes al procedimiento, dada las características fisiológicas y anatómicas de equino, tienen la posibilidad de presentar miopatias post anestésicas algunas de las cuales incluso pueden comprometer la futura aptitud atlética y/o la vida del animal.

Se ha observado que el decúbito dorsal es más perjudicial que el decúbito lateral y que el decúbito lateral izquierdo produce menor alteración que el decúbito lateral derecho.

La ocurrencia de miopatias post anestésicas se asocia a una serie de factores entre ellos; la hipotensión con que cursa una anestesia general inhalatoria, la acción directa e indirecta de las drogas usadas, la posición que adopte el animal durante la cirugía, el peso del animal, las características de acolchamiento de la cama quirúrgica, como también de una posible predisposición genética del ejemplar.
En el diagnostico de esta patología juegan un rol importante las mediciones que se puedan hacer de enzimas séricas tales como, CK, AST y $\mathrm{LDH}$, las cuales actúan como indicadoras de la magnitud de daño del músculo como del tiempo de evolución del cuadro.

No se conocen medidas preventivas de la ocurrencia de miopatias post anestésica, sin embargo, algunos factores pueden disminuir la ocurrencia de ellas. Así, el conocimiento anamnésico de la ocurrencia anterior de alguna forma de este tipo de cuadros, el excluir el grano de la alimentación del animal por lo menos 72 horas antes del procedimiento anestésico, la posición del animal en la cama quirúrgica, el mantener el paciente en un plano anestésico lo mas bajo posible y monitoreando en forma permanente la presión arterial evitando el que curse con hipovolemia, son factores que pueden disminuir el riesgo de ocurrencia de estas miopatias post anestésicas.

\section{REFERENCIAS}

1.- ALEMÁN M. 2005. Equine Neuromuscular Disease. Journal of Equine Veterinary Science 25 (7): 295-299.

2.- ALEMÁN M. 2008.A review of equine muscle disorders. Neuromuscular Disorders 18: 277-287.

3.- BIDWELL L, BRAMALAGE L, ROOD W. 2007. Equine perioperative fatalities associated with general anaesthesia at a private practice- a retrospective case series. Veterinary Anaesthesia and Analgesia 34: 23-30.

4.- COLGAN S, REECE R, HUGHES KJ. 2006. Polysaccharide storage myopathy in an Australian Quarter Horse. Australian Veterinary Journal 84 (12): 436-438.

5.- CORNICK-SEAHORN J. 2004. Anesthesia of the critically ill equine patient. Veterinary Clinics. Equine Practice 20: 127-149.

6.- DE OLIVEIRA A, SANTOS C, REICHMANN P, RODRIGUEZ G, CONSTANTINO M, COSENZA M. 2008. Surgical Management of cecal impaction/ Dysfunction by ileocolostomy in a horse. J. Equine Vet Sci 28 (1): 34-39.

7.- DOHERTY T, VALVERDE A. 2006. Manual of Equine Anesthesia and Analgesia. Primera edición. Oxford, Blackwell Publishing Ltd, $362 \mathrm{p}$.

8.- DUKE T, FILZEK U, READ M, READ E, FERGUSON J. 2006. Clinical observations surrounding an increased incidence of postanesthetic myopathy in halothane-anesthetized horses. Veterinary Anaesthesia and Analgesia 33: 122-127.

9.- GODOY A. 1992. Anestesia general endovenosa en equinos. Monografías de Medicina Veterinaria, Vol. 14 (1).

10.- GOODRICH L, MCILWRAITH C. 2009. Complications Associated with Equine Arthroscopy. Vet Clin Equine 24: 573-589. 
11.- GRINT N, GORVY D, DUGDALE A. 2007. Hyperthermia and Delayed-Onset Myopathy after Recovery from Anesthesia in a Horse. Journal of Equine Veterinary Science 27 (5): 221-227.

12.- GUYTON A, HALL J. Tratado de fisiología médica. Décima edición, 2001, Editorial McGraw-Hill Interamericana, Madrid, España. 1280 pp.

13.- KLEIN L. Anesthetic complications in the horse. The Veterinary Clinics of North America. Equine Practice 1990; 6: 665-692.

14.- LEADON DP. 2006. Haematology and Biochemistry of the equine athlete at rest. Proceedings of the $9^{\text {th }}$ International Congress of World Equine Veterinary Association 291-295.

15.- LEMUS A. 1990. Evaluación de dos protocolos anestésicos en Equinos Fina Sangre de Carrera utilizando halotano y halotano/oxido nitroso en la mantención de la anestesia quirúrgica. Memoria para optar a título profesional de Médico Veterinario. Santiago, Chile. Universidad de Chile, Facultad de Ciencias Veterinarias y Pecuarias, $93 \mathrm{p}$.

16.- LINDSAY WA, ROBINSON GM, BRUNSON DB, MAYORS LJ. 1989. Induction of equine postanesthetic myositis after halothane induced hypotension. Am J Vet Res 50: 404

17.- LUCAS RG, SATUÉ K. 2002. Miopatías en el caballo II: Nutricionales y metabólicas, por alteraciones en la contractibilidad, traumáticas y otras patologías musculares. Medicina Veterinaria 19 (4): 57-70.

18.- MEYER H, KÄSTNER S, BEYERBACH M, REHAGE J. 2009. Cardiopulmonary effects of dorsal recumbency and high-volume caudal epidural anaesthesia with lidocaine or xylazine in calves. The Veterinary Journal, In Press, Corrected Proof, Available online 21 September.

19.- MITCHELL MA, SANDERCOCK DA, HUNTER RR, CARLISLE AJ. 1999. Skeletal muscle damage following halothane anaesthesia in the domestic fowl: plasma biochemical responses. Research in Veterinary Science 67: 59-64.

20.- RAISIS AL. 2005. Skeletal muscle blood flow in anaesthetized horses. Part II: effects of anaesthetics and vasoactive agents. Veterinary Anaesthesia and Analgesia 32: 331-337.

21.- REED SM, BAYLEY WM, SELLON DC. 2004. Equine Internal Medicine. $2^{\mathrm{a}}$ edición. Elsevier (USA), Saunders, $1659 \mathrm{p}$.

22.- RUDOLPH W. 2003. Manual de Bioquímica Clínica Animal. Santiago, Chile. Editado por Wilhelm G. Rudolph Rojas, $97 \mathrm{p}$.

23.- SERTEYN D, MOTTART E, DEBY C, DEBY-DUPONT G, PINCEMAIL J, PHILIPART C, LAMY M. 1990. Equine postanaesthetic myositis: a possible role or free radical generation and membrane lipoperoxidation. Res Vet Sci 48: 42.

24.- SMITH RKW, DYSON S, HEAD M, BUTSON RJ. 1996. Ultrasonography of the equine triceps muscle before and after general anaesthesia and in post anaesthetic myopathy. Equine Veterinary Journal 28 (4): 311-319.

25.- STASHAK T. 2004. Adams: Claudicación en el caballo. Buenos Aires, Inter-Médica, 1248 p.

26.- TIZARD I. 1998. inmunología Veterinaria, $5^{\text {a }}$ edición. Philadelphia, McGraw-Hill Interamericana Editores, $567 \mathrm{p}$.

27.- TRANQUILLI W, THURMON J, GRIMM K. 2007. Lumb \& Jones' Veterinary Anesthesia and Analgesia. $4^{\text {a }}$ edición. Iowa, USA, Blackwell Publishing, 1096 p.

28.- WAGNER A. 2009. Complications in Equine Anesthesia. Vet Clin Equine 24: 735-752.

29.- WHITE N, SUAREZ M. 1986. Changes in triceps muscle intracompartmental pressure with repositioning and padding of the lowermost thoracic limb of the horse, American Journal of Veterinary Research 47: 2257. 